\title{
Éditorial
}

\section{Les pôles régionaux de développement}

Ciblée sur la fusion de municipalités, la récente réforme des agglomérations urbaines a certes amélioré les conditions institutionnelles aptes à soutenir le renforcement des différents pôles du Québec, ne serait-ce que par cette nouvelle capacité collective de projection des efforts de concurrence vers l'extérieur de chaque agglomération plutôt que vers l'intérieur. Cette représentation renouvelée de la compétitivité au sein des pôles québécois de croissance s'inscrit tel un gain important dans le contexte actuel. Car les villescentres sises au cœur des agglomérations québécoises sont en compétition réelle avec Prince-George, Bangor, Oulu, Kingston, Morioka, Kennewick, Luela plutôt qu'avec Moisie, Saint-Lucien, Jonquière, Cap-dela-Madeleine, Pointe-aux-Trembles et Cap Rouge.

En regard de ce renforcement des pôles, d'aucuns considèrent qu'il reste cependant beaucoup de travail à faire. Puisque la recommandation de Roland Parenteau formulée en 1964 demeure toujours sinon encore plus valable à propos de la "nécessité de miser sur certains points intermédiaires de la structure québécoise de peuplement afin d'y favoriser la polarisation, le développement et la diffusion de ses effets ", nul doute que la réforme des agglomérations offre un momentum approprié pour continuer l'œuvre de consolidation des différents points d'ancrage de l'aménagement, de la gestion et du développement des régions au Québec.

Quelques questions sont alors inévitablement soulevées à la réflexion collective. À la lumière des tendances spatiales actuelles, quels sont ces points intermédiaires d'ancrage sur lesquels les autorités du Québec doivent miser pour soutenir certains efforts de développement régional ? Afin d'offrir un éclairage contemporain à cette question, il devient nécessaire, à notre avis, de bien saisir la dynamique géoéconomique propre au Québec. Selon notre lecture, cette géo-économie des pôles régionaux s'inscrit à l'enseigne de cinq tendances spécifiques qui l'influencent, soit les forces spatiales de la compétitivité, les aires de rayonnement des pôles, les systèmes urbains, la rationalisation des points de desserte du secteur public ainsi que le repositionnement relatif déjà en cours de certaines agglomérations. Voyons un peu ces cinq composantes de base sur lesquelles la recherche scientifique pourrait être interpellée davantage.

Les pôles régionaux ne possèdent pas tous les mêmes forces d'attraction des activités humaines. Les différences s'inscrivent dans leur bassin de main-d'œuvre, dans leurs équipements et services publics, dans leur dynamique socioculturelle, etc. Certaines agglomérations sont localisées près d'un important bassin de ressources naturelles, au centre d'une vallée fertile ou dans la frange de la métropole Montréal. D'autres pôles bénéficient de la proximité de la frontière américaine de plus en plus traversée par des échanges, alors que d'autres agglomérations encore possèdent un accès direct à la voie maritime du Saint-Laurent. Ces diverses localisations qui soutiennent certaines forces spécifiques n'offrent aucunement la même attractivité potentielle pour toutes les agglomérations urbaines du Québec. Chaque pôle possède ses propres spécificités sur lesquelles il doit miser adéquatement pour polariser des activités de nature sociale, culturelle, administrative et économique.

La localisation particulière de divers pôles sur l'espace québécois offre aussi à chacun sa propre capacité de rayonnement sur une aire plus ou moins étendue. Les commerces (notamment les Géants de la distribution), les services privés et publics tels que l'éducation supérieure et les groupes-conseils, les bassins d'emploi définis par les migrations alternantes quotidiennes ainsi que la collecte de matières premières par les usines sont autant de dimensions par lesquelles s'exprime ce rayonnement d'un pôle. La distance entre deux centres urbains en concurrence représente 
à cet effet un facteur important de l'étendue de ce rayonnement. À titre d'exemple, Sept-Îles possède évidemment une zone de rayonnement plus vaste que celle de Sherbrooke, mais tout de même moins populeuse. D'une manière générale au Québec, on a constaté, lors d'une enquête récente, que les pôles régionaux de la vallée du Saint-Laurent voient leur aire de rayonnement se stabiliser et même se rétrécir en superficie. Alors que certains pôles plus périphériques comme Saguenay et Rimouski rayonnent sur des aires de plus en plus étendues, quelquefois au-delà même des limites des régions administratives fixées jadis sur la base de ce critère relié au rayonnement effectif.

La position du pôle dans son propre système urbain représente aussi un facteur discriminant sous l'angle de sa capacité de polarisation réelle. À titre d'exemple, la ville de Rouyn-Noranda n'a pas la même importance dans son système urbain abitibien de forme quadri-centrique que Rimouski dans sa Gaspésie dont la structure de peuplement s'avère plutôt monocentrique, même s'il existe plusieurs petits pôles dynamiques comme Carleton, Gaspé, Bonaventure. Trois-Rivières et Saguenay, par contre, doivent partager leur capacité de polarisation avec un autre pôle régional limitrophe, soit Shawinigan et Alma. Alors que Sherbrooke et Gatineau s'avèrent soumis à beaucoup moins de concurrence immédiate dans leur volonté de polarisation. Tous les points intermédiaires de la structure québécoise de peuplement ne possèdent certes pas le même potentiel de polarisation et de diffusion du développement. Des choix dans le soutien public seraient souhaitables.

Une autre dimension qui génère des effets considérables en matière de polarisation s'inscrit dans la masse critique de services publics présents au sein d'une agglomération urbaine. Certains pôles offrent en effet un ensemble de biens et de services publics plus intéressants que d'autres, notamment aux chapitres de l'éducation supérieure, de la R\&D, des activités du tertiaire moteur. À cet égard, la rationalisation dans le secteur public, qui implique des coupures dans les agences régionales des ministères, affecte négativement davantage certains pôles plutôt que d'autres. Drummondville, Joliette, Saint-Georges et Rivièredu-Loup s'en sortent mieux à cette rubrique puisque leur rôle de capitale régionale est en progrès constant sous l'angle des effectifs. Par contre, plusieurs pôles régionaux voient s'effriter progressivement le nombre réel de ressources humaines actives au total dans les directions, bureaux, conseils, centres et autres agences gouvernementales.

Finalement, on constate que certaines agglomérations régionales polarisent globalement plus que d'autres sous l'angle de l'emploi. Pour diverses raisons reliées notamment aux forces spatiales à l'œuvre, à l'aire effective de rayonnement, au type de système urbain, au rôle réel dans la desserte publique de biens et services ainsi que bien d'autres facteurs tels que l'entrepreneuriat et les créneaux porteurs, les pôles régionaux se sont clairement différenciés, entre 1986 et 2001, dans leur capacité de repositionnement sur l'échiquier québécois. À titre d'exemple, la polarisation sous l'angle de l'emploi s'avère très forte à SaintGeorges, Victoriaville, Drummondville et, dans une moindre mesure, à Rivière-du-Loup et à Grandby, alors que cet indicateur décline ou stagne à Shawinigan, Baie-Comeau, Saguenay et Lachute. Il faut prendre acte de cette polarisation très inégale de l'emploi et tirer les recommandations qui s'imposent pour le pouvoir public.

Devant cette variété de facteurs qui distinguent les divers pôles régionaux du Québec, on comprend qu'une stratégie gouvernementale visant à les renforcer doive bien saisir les enjeux pour chaque agglomération. Si l'urbanisation diffuse en zones périurbaines doit être généralement mieux maîtrisée, d'autres interventions reliées aux économies d'agglomération, au marketing territorial, au tertiaire moteur, à l'innovation, au système de transport doivent être adaptées aux forces, aux faiblesses et aux options d'actions de chaque pôle régional. Dans cet esprit, davantage de connaissances pourrait à notre avis converger vers une meilleure modélisation de la dynamique géo-économique propre au Québec dans son contexte nord-américain. En outre, la centaine de petits pôles ruraux qui agissent positivement dans la consolidation de la structure de peuplement périphérique du Québec ne doivent surtout pas être oubliés.

\section{Marc-Vrbain Proulx}

Le Directeur 